\title{
Pitong Sulyap sa Pilosopiya ng Wika ni Padre Ferriols
}

\section{Wilhelm Patrick Joseph S. Strebel}

\begin{abstract}
The Jesuit priest, Roque J. Ferriols is well known in Ateneo de Manila University as the first philosophy professor to teach philosophy in Filipino. Since 1969 until he retired in 2014, he taught all of his philosophy classes, both in the undergraduate and graduate levels, in Filipino. His example was followed by many Ateneo philosophy faculty. Since around the 1990s the number of philosophy classes taught in English and in Filipino had a 50/50 percentage ratio. This ratio continued on to the present time. As the tradition of teaching philosophy in Filipino nears its 50 th anniversary in 2019, Ferriols continues to be appreciated as the trailblazer of the tradition. Even if Ferriols never taught philosophy of language, his teaching pedagogy and his writings reflect important insights relevant to the philosophy of language. This paper seeks to draw these insights from three different texts written years apart from each other but all within Ferriols's teaching career. The texts are A Memoir of Six Years, an essay written for Philippine Studies in April 1975; Sinauna Bilang Potensyal, the final chapter of his Pambungad sa Metapisika published in 1991; and a few sections of his memoirs, Sulyap sa Aking Pinanggalingan published in 2016. In so doing, this paper seeks to spearhead critical discussions of Ferriols's philosophy of language stressing on how these insights come from the context of pedagogy and as such how these insights reflect the context, experience, lifeworld and historicity of the learner towards a deeper understanding of the human being qua linguistic being and human being qua human being. Thus, the paper seeks to show that the conscious and deliberate awareness of language and culture often seen in the celebration of Buwan ng Wika distracts us from the reality and nature of language and culture, and obscures the role of language and culture in human life. The main emphasis of this paper is the insight that the reality, nature, and role of language and culture could be found in the actual lived experience of the world as linguistic reality. In other words, we do not experience the world "through the medium" of language and culture. What really happens is that our experience of the world is language and culture. This paper does not pretend to be exhaustive in that it cannot fully follow the logical and
\end{abstract}

(C) 2018 Wilhelm Patrick Joseph S. Strebel

https://www.kritike.org/journal/issue 22/strebel june2018.pdf

ISSN 1908-7330

(cc) BY-NC-ND 


\title{
40 PILOSOPIYA NG WIKA NI FERRIOLS
}

\begin{abstract}
phenomenological implications of the insights it brings to the reader's attention. Its task is to make thematic these insights and note that these insights are conscious to Ferriols as a philosophy teacher so that these themes and insights could be further mulled over and reflected upon by other writers and scholars seeking to elucidate on the subject matter.
\end{abstract}

Keywords: Filipino Philosophy, Philosophy of Language, Philosophy in Filipino, Language and Pedagogy

\section{Ang Kontekstong Historikal}

S usi sa pag-uunawa ng kultura, lipunan, at danas-daigdig nating mga Pilipino ang katunayang nasakop ng mga kanluraning kapangyarihan ang ating bansa. Ang pagkadominante ng relihiyong Kristiyano sa kapuluan ay bunga ng 300 taong pananakop ng mga Kastila. Ang pananaig ng Ingles bilang wika ng mga institusyon ng edukasyon, at ng mga pangunahing anyo ng pagpapahayag tulad ng radyo, telebisyon, at pati ng internet ay bunga ng mga limang dekada ng pamamalakad ng mga Amerikano sa bansa. Iniulat ni Ferriols na noong dekada '60, makadarama ang sinumang nakatira sa Kamaynilaan na mayroong namamayaning diwa na makapiling ang wikang Filipino. ${ }^{1}$ Ibig niyang sabihin na nagsimula nang magnais ang mga Pilipino na sambitin at pakinggan ang sariling wika. Sa mga talakayan ng mga pulitiko pati ang pag-uulat sa radyo maraming nagsikap bigkasin ang wikang Filipino. Ang sentimyentong ito ang siyang matatawag nating Filipinization: bagaman may pananaig ng Inggles, may tumutubong pagnanais na manahan sa Filipino. Isang halimbawa nito sa kulturang popular ay ang simulain ng 'Original Pilipino Music' (OPM) kung saan nagkasundo ang ilang mga Pilipinong kompositor, mangangawit at manunugtog na magsulat, magtanghal, at magsagawa ng recording ng mga awitin sa wikang Filipino.

Nadama rin ang ihip ng hangin ng Filipinization sa Pamantasang Ateneo de Manila, isang pamantasang itinatag ng mga Heswitang Kastila noong 1853. Sa panahon ng mga Amerikano, nalipat sa pamamalakad ng New York Province ng mga Heswita ang pamantasan kung kaya't hanggang sa katapusan ng ikalawang milenyo malimit ang presensya ng mga Heswitang Amerikano sa mga bulwagan at silid ng pamantasan. Dito malamang galing ang pananaw ng maraming tao ukol sa Ateneo twang: ang

${ }^{1}$ Tingnan ang Roque J. Ferriols, "A Memoir of Six Years," sa Pagdiriwang sa Meron: A Festival of Thought Celebrating Roque J. Ferriols, S.J., ed. by Nemesio Que and Agustin Martin G. Rodriguez (Quezon City: Office of Research and Publications, Ateneo de Manila University, 1997), 215. Sa mga susunod na pagkakataon, tutukuyin ang akdang ito bilang A Memoir.

(C) 2018 Wilhelm Patrick Joseph S. Strebel

https://www.kritike.org/journal/issue 22/strebel june2018.pdf

ISSN 1908-7330

(cc) BY-NC-ND 
pamamaraan ng pagsasalita ng mga Atenista ng wikang Ingles at pati ng wikang Filipino na may puntong New York.

Nagsimulang magturo si Ferriols ng pilosopiya sa Ateneo de Manila noong 1960. Pagpasok ng akademikong taon 1969-1970, nagsimula siyang magturo ng pilosopiya sa wikang Filipino. Iniulat niya na hindi naging madali ang kanyang pagpapasimuno. Humarap siya sa maraming tahasang batikos at pati tahimik na pagtanggi mula sa ilang kasapi ng administrasyon at ng mga kapwa guro. Ang ilan sa kanila'y mga Amerikano ngunit may ilan din sa mga ito na Pilipino. Bagaman malamig ang suporta ng administrayon (tinawag na eksperimental ang mga klase niya at ibinigay sa kanya ang mga pinakamaaaga at pinakahuling oras pati rin ang oras ng tanghalian para magklase: mga oras na hindi ituturing na ideyal para sa pagkaklase), itinuring niyang biyaya na buo ang loob ng mga estudyante niyang dumalo sa kanilang mga klase.

Hindi niya masisisi ang mga Amerikanong administrador at guro ng pamantasan ngunit ang ipinagtaka niya ay ang pananaw ng ilang mga kapwa niya Pilipinong guro ukol sa kaniyang naging kilos. Tila baga kumbinsido ang mga taong ito na walang kakayahan ni karapatan ang wikang Filipino na maging wika ng pag-iisip, pag-aaral, pagtuturo, pananaliksik, pakikipagtalastasan, at iba pa. May mga hindi makapaniwala, nagmata, at kumutya sa kanyang naging kilos.

Sa mga taong lumipas, marami nang nangyari ngunit, hindi pa rin nagbago ang mga kalagayan. Bagaman sa kasalukuyan ay may polisiyang baylingwal ang pamantasan at sinasabi nitong, ang Ateneo ay isang pamantasang Filipino, ang Kagawaran ng Pilosopiya lamang ang may balanseng bilang ng mga klase sa Ingles at sa Filipino. May ilan din namang kursong naituturo sa Filipino tulad halimbawa ng teolohiya at agham pampulitika ngunit nakadepende ito sa guro at hindi institusyonalisado tulad nang sa pilosopiya.

Bukod dito, nananatili pa rin ang pagtanggi ng maraming mga estudyante kung sakaling maubusan sila ng klase sa Ingles at mapilitan silang kumuha ng klase sa Filipino. Kahit lumaki sila sa Kamaynilaan, Calabarzon o Mimaropa, igigiit pa rin nila na hindi sila marunong at hindi sila makapagiisip sa wikang Filipino. Pumasa din naman sila sa mga kursong kinuha nila mula sa Kagawaran ng Filipino, ngunit, mas pipiliin pa rin nilang mag-aral sa Ingles kaysa sa Filipino.

Kung sa bagay, hindi na rin dapat maging kagulat-gulat ito sapagkat nangangailangan pa ng mas malawak na kamulatan ang pag-uunawa ng mga Pilipino ukol sa kultura at wika. Makikita ito sa pagdaos ng Buwan ng Wika tuwing Agosto. Naglalaan tayo ng isang buwan upang isuot ang mga tradisyunal na baro at kumain ng mga kakanin. Para bagang ang pagiging Pilipino ay nakasalalay sa pag-uunawa natin sa anumang itinatawag nating 


\section{PILOSOPIYA NG WIKA NI FERRIOLS}

Filipiniana. Kung susulyapan nga naman ito muli, katwa rin na mayroon tayong Buwan ng Wika. Kung kailangan pang ituro sa mga bata kung paano pahalagahan ang sarili nilang kultura at wika, ibig sabihin lamang nito na walang intrinsikong pagpapahalaga ang mga bata sa sarili nilang kultura at wika. Sa madaling salita, naglalaan tayo ng isang buwan upang pansinin ang sarili nating kultura at wika habang may labing-isang buwan tayo upang baliwalain ito.

Sinusuri sa pambungad na ito ang pag-uugali at pananaw sa ilalim ng pagdiwang ng Buwan ng Wika upang ipakita ang pananaw na kailangan natin ng mulat na pagtataguyod at sadyang pagsusulong ng wika at kultura. Lilitaw sa papel na ito na ang tunay na diwa ng wika at kultura ay naroon sa hindi sinasadya at hindi kadalasang pinapansin na pagpasok at pagdanas ng tao sa kayamanan ng sarili at ng daigdig. Hindi natin dinaranas ang daigdig "sa pamamagitan" ng wika at kultura, bagkus ang wika at kultura ang mismong pagdanas natin sa daigdig. Ito ang pangunahing puntong nais ipakita sa pagsulyap sa pilosopiya ng wika ni Ferriols.

\section{Ang Mga Tekstong Susulyapan}

Ang mga sumusunod ang mga teksto ni Ferriols na hahanguan natin ng kanyang pilosopiya ng wika. Magbibigay muna ako ng pagsasakonteksto para sa bawa't isa.

Isinulat niya ang A Memoir of Six Years noong Abril 1975, anim na taon mula nang simulan niyang magturo ng pilosopiya sa wikang Filipino. Binalik-tanawan niya ang kanyang mga naranasan sa mga taong iyon upang maglabas ng isang apologia, o isang manifesto: alang-alang sa mga hindi nakauunawa kung bakit siya nagtuturo ng pilosopiya sa wikang Filipino. Habang tinutugunan niya ang pitong mga tanong na madalas naibato sa kanya sa nakaraang anim na taon, nakapagbigay-linaw din siya sa kalikasan ng pilosopiya, sa kalikasan ng wika, at sa kalikasan ng tao bilang nilalang na nagsasalita.

Dumaan pa ang dalawang dekada bago nailimbag ang aklat ni Ferriols para sa kanyang klase sa pilosopiya ng tao. Samakatwid, mahabang panahon din siyang gumamit ng mga tekstong Ingles na sinabayan niya ng mga nota niya sa Filipino para sa mga estudyante niya sa mga taong iyon. Noong 1991, lumabas ang Pambungad sa Metapisika. Upang lagumin ang mga puntong naisaad sa aklat na ito, tinutukan niya ang paksa ng potensyal sa huling yugto nito. Naidiin niya ang halaga ng potensyal ng wika sa paggising ng tao sa kalaliman ng meron na siyang nararanasan sa pakikipagkapwa sa kapwa tao, sa daigdig, sa Maykapal, sa sangkameronan. Ito ang yugtong pinamagatang Sinauna Bilang Potensyal.

(c) 2018 Wilhelm Patrick Joseph S. Strebel

https://www.kritike.org/journal/issue 22/strebel june2018.pdf

ISSN 1908-7330

(cc) BY-NC-ND 
Hahango rin ang sulating ito mula sa Sulyap Sa Aking Pinanggalingan, ang aklat na bunga ng pagbibigay-loob ni Genesis John M. Martinez, isang caregiver na idikta sa kanya ni Ferriols ang mga alaala nito upang maipaskil sa isang $b \log$. Nasimulang isulat ito noong 2012 at nalimbag ang aklat noong 2016. Sa panahon ng pagkakasulat ng Sulyap nagpasya si Ferriols na magretiro na sa pagtuturo.

\section{Pitong Sulyap sa Pilosopiya ng Wika ni Ferriols}

Hindi nagturo ng kursong pilosopiya ng wika si Ferriols ngunit mahihinuha sa kanyang pamamaraan ng pagtuturo ang ilang mga malalalim na pagmumulat at pag-uunawa sa kalikasan ng wika. Nais ngayon ng papel na ito palitawin at bigyan ng balangkas ang naturang pagmumulat upang makapagtukoy ng ilang mga direksyong maaaring tahakin sa patuloy na pagtalakay sa paksang napili. Magbibigay tayo ngayon ng pitong landas.

\subsection{Pilosopiyang Filipino}

May malaking kaibahan sa pagitan ng Pilosopiyang Filipino at pamimilosopiya sa Filipino. Ang una ay nakatutok sa tatak, kung saan nagiging abala ang tao sa tanong hinggil sa identidad kung kaya't natatanggal ang kanyang pansin sa mismong akto ng pamimilosopiya. Ang huli naman ay ang pagsusumubok mamilosopiya sa katutubong wika tulad ng maraming pilosopo sa kasaysayan ng sangkatauhan.

Naninindigan si Ferriols na walang Pilosopiyang Filipino. Mahalagang idagdag agad na naninindigan din siya na hindi mahalaga ang mga bansag tulad ng Griyegong Pilosopiya, Pilosopiyang Tsino, o Pilosopiyang anuman. Ang mga pilosopo ay basta lamang namilosopiya na walang intensyong magpasimuno ng mababansagang Pilosopiyang anuman. Ang mga bansag na ito ay mahalaga lamang para sa mga pangangailangan ng kategorisasyon; kategorisasyong mahalaga para sa mga nangangasiwa sa mga silid aklatan, sa tanggapan ng mga registrar ng mga paaralan at ng iba pang mga beyurokratikong pangangailangan. Ang punto ni Ferriols ay lilinaw kung pag-uusapan bilang analohiya ang tinatawag na mga genre o uri ng musika. Ang musikero ay abala sa paglikha ng musika. Kung anuman ang itatawag dito ng madla ay hindi na niya pagkakaabalahan. Ang mga bansag kung ang musika niya ay alternatibo, OPM, rock, ballad, o anuman ay pangangailangan ng mga negosyante sa industriya ng musika kung paano nila ipakikilala sa publiko ang obra ng musikero. Ang mga tao sa likod ng YouTube, Spotify, iTunes at iba pa ang may pangangailangan ng malilinaw na mga bansag. Sa madaling salita, ang musika ay dinaranas ng mga tumutugtog at nakikinig. Hindi pangunahing pangangailangan ang 


\section{PILOSOPIYA NG WIKA NI FERRIOLS}

pagbabansag dito. At ganoon din ang akto ng pamimilosopiya. Ito ay dinaranas at ginagawa. Oras na tanungin ng namimilosopiya kung nagpapakapilipino pa ba siya, nadidiskaril sa ibang paksa ang daloy ng kanyang pag-iisip.

Kung tutuusin, ang unang puntong ito ay walang kinalaman sa pilosopiya ng wika ngunit mahalagang banggitin upang maisantabi. Sa paglilinaw kung ano ang hindi ginagalawan ng pilosopiya ng wika, harinawa magbigay-linaw ito, sa di-tuwirang paraan, kung ano ang tunay na ginagalawan nito.

\subsection{Ang Wika Bilang Di-Makatawag-Pansin-sa-Sarili}

Tuwing tinatanong si Ferriols ukol sa kanyang pangangahas na mamilosopiya sa wikang Filipino, palagi niyang idinidiin na ang tunay na pakay ay ang mamilosopiya at hindi ang anumang pulitikal, lingwistiko o kultural na dahilan. Ang hamon ay hindi ang hamon ng pagbigkas ng anumang wika. Bagkus, ang tunay na hamon ay ang hamon ng pilosopiya at ang hamon ng akto ng pamimilosopiya.

Ito ang tugon niya sa mga nagtatanong kung nais ba niyang isulong ang Tagalog bilang wikang pambansa. At siyempre, sangkot sa tanong ang ilang mga pulitikal at kultural na mga agenda na may mulat na naisin ukol sa kung paano ba dapat isinasagawa ang mga sistema ng edukasyon at ng media. May isang buong larangan ng pagpapahalaga kung saan maaaring magdiskurso ukol dito ngunit hindi ito ang pakay ni Ferriols.

Hinggil sa Tagalog, mahalagang ipunto na marami itong anyo. May Tagalog Bulakan, Tagalog Kabite, pati Tagalog Marikina. Aling Tagalog nga kaya ang tinutukoy natin? Muli, nasa labas ng pinagkaka-abalahan ni Ferriols ang mga tanong na ito. Mas payak dito ang kanyang tunay na dahilan ng pagtuturo sa wikang Filipino: sapagkat nasa lungsod ng Quezon ang Ateneo de Manila kung saan siya nagtuturo, nais niyang magturo ng pilosopiya at matutong mamilosopiya ang kanyang mga estudyante sa wika ng naturang lugar. Bago pa siya madestino sa Ateneo de Manila, nagturo din siya noon sa Berchmans College sa Cebu. Kung naiba lang ang kasaysayan at nalagi siya sa Cebu, malamang ang maipapasimuno niya ay ang pagtuturo ng pilosopiya sa wikang Cebuano.

Dagdag pa rito, sinasabi niyang hindi niya paboritong wika ang Filipino, ngunit mahusay naman din itong wika. Sa madaling salita, ang tunay na dahilan ng pagbabad sa wikang Filipino ay ang halaga ng pagbabad sa danas-daigdig ng estudyante at ng lokalidad ng paaralan. Hindi ang wika o ang wikang Filipino ang sentro ng usapan. Nalilihis ang atensyon ng pumupuna kung papansinin niya ang wika imbes na danasin ang daigdig ng kahulugan na nagpapakita sa wika. Hindi tumatawag ng pansin sa kanyang 
sarili ang wika. Kapiling natin, sapagkat palagi tayong bumibigkas. Nasa sentro ng lahat ng usapin ngunit hindi nagiging sentro ng usapan, maliban lang kung punahin ng pilosopo sa kanyang pagmumuni.

\subsection{Ang Wika bilang Paraan ng Pagiging Buháy}

Ang wika ay hindi lamang isang kasangkapang ginagamit ng nagsasalita sa kanyang pangangailangang ipahayag ang kanyang sarili upang maunawaan siya ng kapwa tao. Nanganganib ang wika sa ganitong simplistikong pananaw lalo na't ginagamit sa mga institusyon ng edukasyon ang konsepto ng medium of instruction. Para bagang iniisip ng ganitong kalakaran na ang nilalaman ng isang kursong itinuturo ay maaaring itawid ng guro sa estudyante sa anumang wika sapagkat bilang medium (Latin: gitna o pagitan), ito ang tulay sa pagitan ng guro at estudyante. Ang mahalaga ay ang itinatawid na nilalaman o kaalaman. Ang wika ay instrumento lamang na nagbibigay-daan sa naturang pagtawid. Ganoon lang ba?

Sa halip, natuklasan ni Ferriols na ang wika ay hindi lamang paraan ng pagpapahayag tulad ng kaisipan ng medium. Lagpas pa rito at lalong mahalaga pa, ang wika ay isang paraan ng pagiging buháy. Wika nga niya:

Ang siyang makasalíng sa pinakapuso ng isang wika, kahit sa isang kisap-mata lamang, nalalaman niyang ang wika ay isang di-mahahangganang paraan ng pagiging buháy. May di-mauulit na potensyal ang bawat wika sa pagtingin at pagdama; sarili nitong talino, sarili nitong dating. Kung mas marami kang wikang nadarama, mas buháy ka. ${ }^{2}$

Ipakita natin gamit ang isang halimbawa. Hindi lihim ang pagpapahalaga ng mga Pilipino sa pakikipagkapwa-tao. Ang pagpapahalagang ito ay nababakasan sa wika mismo. Malawak at inklusibo ang pagmumulat ng Pilipino sa kung sino ang kapamilya. Kung kaya't may partikular siyang tawag sa mga kapamilya niyang dulot ng ugnayang kasal. Bukod sa mga partikular na katawagang 'biyenan,' 'manugang,' 'bayaw,' at 'hipag,' kung saan ang mga katumbas sa Ingles ay mga salitang dinugtungan lamang ng in-law, may mga salitang ugnayang hindi maisasalin sa mga kanluraning wika. Ang 'bilas' (asawa ng kapatid ng asawa ko) at 'balae' (ang mga magulang ng manugang $\mathrm{ko}$ ) ay mga salitang walang salin sa wikang Ingles. Nabubuhay ang Pilipino nang may pagkilala sa mga ugnayang ito. Sa ganitong paraan nabubuhay ang mga Pilipino. Hindi niya, malamang,

${ }^{2}$ Ferriols, A Memoir, 217. Akin ang salin.

(c) 2018 Wilhelm Patrick Joseph S. Strebel https://www.kritike.org/journal/issue 22/strebel june2018.pdf ISSN 1908-7330 


\section{PILOSOPIYA NG WIKA NI FERRIOLS}

napapansin hangga't hindi niya nakakatagpo ang ibang mga wika. Sa pagmumulat ko sa mga salita ng aking wika, lalo akong nagiging mulat kung paano ako nabubuhay. Ang bilas ko at ang balae ko ay itinuturing kong may partikular na kaugnayan sa akin. Sa taong nabubuhay sa ibang wika, ituturing lamang sila bilang mga kamag-anak ng sariling asawa at hindi nauugnay nang ganoon sa kanyang sarili.

Mapapansin din sa huling bahagi ng sinipi mula sa itaas na tinukoy ni Ferriols ang pagdama imbis na pag-alam sa wika. Karaniwang sinasabi na ang wika ay inaalam, natututunan at umaabot ang tao sa punto kung saan masasabing marunong na siyang magsalita. Ngunit ang sabi ni Ferriols ay “kung mas marami kang wikang nadarama, mas buháy ka." Ang wika ay hindi lamang inaalam, bagkus dinarama. At ang kakayahang makadama ay ang mabuhay nang totoo. Makatutulong ang kwento ni Ferriols ukol sa pons asinorum (Latin: tulay ng mga asno). ${ }^{3}$ Ang natututong magsalita, maging batang maliit o matandang nais matuto ng bagong wika ay parang asnong may mahahabang tainga sa simula. Habang natututo siyang pagsikapan ang wika, para siyang asnong sumusubok tumawid ng tulay ng pag-uunawa. Mabagal at mahirap ang landas ngunit kung magpupursigi siya na parang asno, makatatawid din siya sa tulay, mahuhulog ang mga tainga niyang mahahaba at magugulat na lamang siya na isa na siyang taong naka-uunawa at naka-sasalita.

Para sa taong nag-aaral ng bagong wika, nakatutulong ang pag-aaral ng gramatika ng wikang pinag-aaralan. Ganito ang hamon ng pagtawid ng pons asinorum para sa nag-aaral ng bagong wika. Maaari siyang magulumihan, mabagot, mahirapan, mamilipit sapagkat iba ang balangkas ng gramatika ng bagong wika sa gramatika ng wikang kilala na niya. Kailangan niyang magtiis. Maari siyang malunod sa mga istraktura ng gramatika at hindi na madanas at maunawaan ang sinasabi talaga sa wikang iyon. Ngunit kung magsisikap nga na parang pagsisikap ng isang asno, makatatawid din ang nais makadama at makaunawa.

Sa Sulyap sa Aking Pinanggalingan, ikinuwento ni Ferriols kung paano siya natuto ng Griyego at Latin bilang bahagi ng kanyang pagsasanay sa nobisyado. Habang nasa kadiliman ng Ikalawang Digmaang Pandaigdig, naranasan ni Ferriols ang liwanag na hatid ng pagkakilala niya sa wika ng mga sinaunang Griyego, ang gramatika ng wikang Latin, at ang panitikan ng wikang Ingles. Nagbigay-pugay at pasasalamat si Ferriols sa mga guro niya tulad nina Horacio Dela Costa, Joseph Mulry at Joseph Kerr. Natuto siya at nagising sa kayamanan ng wika sa tiyaga at pagpupursige ng mga gurong ito. Natatanging banggit ang kanyang pasasalamat kay Kerr sapagkat kung

3 Tingnan ang Roque J. Ferriols, Sulyap sa Aking Pinanggalingan, ed. by Leovino Ma. Garcia (Quezon City: Bughaw, Ateneo de Manila University Press, 2016), 95. Sa mga susunod na pagkakataon, tutukuyin ang akdang ito bilang Sulyap.

(c) 2018 Wilhelm Patrick Joseph S. Strebel

https://www.kritike.org/journal/issue 22/strebel june2018.pdf

ISSN 1908-7330

(cc) BY-NC-ND 
hindi siya tinuruan ni Kerr mag-Griyego, hindi niya maisasalin sina Platon, at ang mga pilosopong Griyego. Sapagkat maraming estudyanteng dumaan sa mga klase ni Ferriols sa pilosopiya ng mga sinaunang Griyego, utang na loob din ito ng mga estudyante niya sa mga dating mga gurong hindi na nila nakilala.

Bilang paraan ng pagiging buhay, makararating tayo sa bagong pagmumulat kung paano tayo nabubuhay kung susuriin natin ang katagang 'ka,' na tanda ng ugnayan. Ani Ferriols:

Sapagkat malalim ang hiwaga ng tao, malalim rin ang kanyang pakikibuklod. Tingnan ang katagang kapwa na palaging ginagamit at ang $k a$ na tanda ng pakikipagkapuwa. Mababakasan iyang lahat, at higit pa riyan sa ating samu't saring wika. Ang lahat niyan ay potensyal. Maaari nating buhayin, likhain muli. Maaari nating gawing bahagi ng mga hindi inaakalang kombinasyon. Halimbawa, magawa natin ang hindi pa nagagawa: kalinangan na personal at makatao, at sabay teknikal. Teknolohiya dahil sa tao; at huwag baliktad. ${ }^{4}$

Ang samu't saring wikang tinutukoy ni Ferriols ay ang mga wika sa Pilipinas na siya namang may hawig na oryentasyon. Kung sakaling kaliligtaan at lilimutin ng isang Pilipino, may paraan ng pagiging buhay na mawawala sa kanya, lilimutin niya rin at hihiwalayan; masasayang ang potensyal. At mula sa natuklasan niyang katutubo at likas na pagkiling sa pakiki-ugnay, binibigkas ni Ferriols ang hamon at tungkulin ng kasalukuyang tao sa harap ng kasalukuyang teknolohiya. Kung tumutungo ang kilos ng teknolohiya sa paghihiwalay sa ugnayan, ano ang tugon ng wika dito?

Sa naunang panahon, natutong makihalubilo ang mga bata sa paglalaro ng tumbang preso, patintero, habulan, taguan at iba pa. Ginagawa ito noon ng magkakapitbahay sa lansangan sa labas ng bahay. Sa kasalukuyang mga antas na middle class pataas, madalang na ang ganitong mga pagkakataon. Kanya-kanya na ng gadget at video game ang mga bata kung kaya't kahit mayroon mang network gaming kung saan maaari kang makilaro sa iba online, hindi pa rin nito natutumbasan ang nagaganap na pakikihalubilo, pakikipagsapalaran, at pakikipag-kaibigang ginagalawan ng mga batang naglalaro sa lansangan. Nakikita ni Ferriols ang panganib sa ugnayan at sa buhay kung hahayaan lamang nating tayo ay pangunahan ng teknolohiya sa halip na tayo ang mamahala sa teknolohiya.

${ }^{4}$ Roque J. Ferriols, Pambungad sa Metapisika (Quezon City: Office of Research and Publications, Ateneo de Manila University, 1991), 240.

(c) 2018 Wilhelm Patrick Joseph S. Strebel https://www.kritike.org/journal/issue 22/strebel june2018.pdf ISSN 1908-7330 
PILOSOPIYA NG WIKA NI FERRIOLS

\subsection{Ang Wika bilang Potensyal ng mas Matinding Ugnayan}

Matagal nang bintang sa pilosopiya ang pagiging hiwalay sa daigdig at realidad na nais nitong unawain. Mula nang unang naitampok sa dula ni Aristophanes ang isang tinatawag na Sokrates na naglalambitin sa kaitaasan at pinagsasasabi na lumalakad daw siya sa himpapawid at namumulastog pa ng kung anu-anong kabulastugan, hanggang sa pananaw sa pilosopo bilang taong naglalaro lamang ng mga salita at nakatira sa itaas ng toreng garing.

Mahuhulog sa ganitong panganib ang taong magsasabi na parepareho lamang ang bisa ng mga wika sa paghahanap sa katotohanan, sa pamimilosopiya. Kung kaya't kahit anong wika ay maaaring galawan ng akto ng pamimilosopiya. Hindi na raw mahalaga kung alin. Malimit na nalilimutan sa ganitong puna ang mahalagang katotohanan na palaging napaliligiran ng kapwa tao ang pilosopo. Sumasapanahon at sumasakasaysayan din ang pilosopo. Nakapasok ang kanyang pagmumunimuni sa konteksto ng kanyang pag-iral sa daigdig. At kasama sa konteksto niya ang wika ng pamayanang kinabibilangan niya. Ani Ferriols:

Kung may tao sa aklatan, at sinusubukan niyang mamilosopiya sa isang wika na ibang di hamak sa sinasalita ng mga nagmamaneho ng dyipni, nagwawalis-tingting sa mga kalsada, nagsisilbi sa mga turo-turo, masasabi kaya na ang taong iyon ay gumagalaw sa katotohanan ${ }^{5}$

Lumilitaw na ang tao ngang ganito ang siyang nagbibigay ng masamang reputasyon sa pilosopiya bilang hiwalay sa totoong buhay. Walang ibang daigdig na ginagalawan ang namimilosopiya. Nasa parehong daigdig siya ng kapwa tao. Sa pagbuo niya ng mga abstraksyo, hindi siya nagpapakomportable sa mga abstraksyo. Kung iniiwan man niya ang tunay na buhay upang atupagin ang pagmumuni-muni, ginagawa lamang niya ito upang pasukin nang mas malalim, hindi ang abstraksyo, bagkus ang tunay na buhay.

Maaring mangyari na nasa paliparan ang isang tao. Napapaligiran siya ng mga taong samu't sari ang wika. Sabihin na nating sa tabi niya ay may mga kapwa biyaherong nagkukuwentuhan sa wikang Ruso. Nasa iisang lugar sila ngunit ang mga nakauunawa lamang ng wikang Ruso ang nasa parehong daigdig ng taong nagsasalita. Bawat wika na maririnig sa tanggapan ng paliparan ay isang sariling daigdig na hiwalay sa iba pang daigdig ng iba pang wika. Samakatwid, humihiwalay sa kapwa tao ang taong

${ }^{5}$ Ibid., 236.

(c) 2018 Wilhelm Patrick Joseph S. Strebel https://www.kritike.org/journal/issue 22/strebel june2018.pdf ISSN 1908-7330

(c) BY-NC-ND 
namimilospiya sa wikang iba sa daigdig ng kapwa tao. Patuloy pa nga ni Ferriols:

At kapag ang nagsisikap mamilosopiya ay pumipili sa wikang gagamitin niya, ang kanyang pagpili ay bunga ng kanyang atitud sa salita ng mga pumapaligid sa kanya. At ang kanyang atitud ay maaring katotohanan, maaring kasinungalingan. ${ }^{6}$

Ang pasyang mamilosopiya sa wika ng mundong ginagalawan ng tao ay tugon sa panawagan tungo sa mas malalim na pakiki-ugnay. Sa ganitong paraan nagigisnan ng tao ang wika bilang potensyal ng ugnayan at punto ng hugpungan ng samu't saring tao. Ang pasyang mamilosopiya sa wika ng mundong ginagalawan ay isang pag-amin na nasa iisa at pareho tayong daigdig. Iisa ang ating tahanan, dangal, at larangan. Tao ako sa harap mo at tao ka sa harap ko. Naghaharapan at nagkaka-unawaan tayo bilang magkapatid sa katauhan. Wika ni Ferriols:

Ang huwaran ng ating kalinangan ay tao bilang malalim, sagrado, mapaglikha at nakikipagkapwa sa kapwa-tao at sa Maykapal. Personal na pagbubuklod ng tao sa tao, at personal na pakikisalamuha sa atin ng Maykapal ang buod ng sanlibutan. Nararanasan natin ang meron bilang ugnayan. ${ }^{7}$

Ikinuwento ni Ferriols kung paano siya tinuruan ni Mulry ng panitikan sa nobisyado. Naranasan niya, sa tulong ni Mulry, na ang pagbasa ng panitikang banyaga ay paraan upang maging pamilyar ang daigdig ng mga taong iba sa atin at dahil dito, mabigyan tayo ng pagkakataong makidama o makiramay sa kanila. Noong minsan na nagbasa sila ng isang tula ukol sa mga kalsada ng London, ganito ang nadama niya:

Ito ay ukol sa buhay ng mga tao sa London. Hindi namin sila kilala. At ang kanilang uri ng pamumuhay ay dayuhan sa amin. Pero kaya naming maranasan na talagang may lugar na London at talagang may mga taong nakatira doon. At kahit na ang tula ay isinulat bago magkagiyera alam namin na habang naririnig

${ }^{6}$ Ibid., 236.

${ }^{7}$ Ibid., 239. 


\section{PILOSOPIYA NG WIKA NI FERRIOLS}

namin ang tula, ang mga taong iyon ay nagtitiis din sa giyera. At kaagad nakikiramdam kami. ${ }^{8}$

Tunay ngang potensyal tungo sa ugnayan ang wika. Nabubuksan para sa tao ang dayuhang daigdig at nagiging bahagi ito ng sariling danas-daigdig. Lagpas sa mga anyo at istruktura ng mga tula at ng iba pang mga anyo ng panitikan, idinidiin ni Ferriols na ang panitikan sa partikular at ang wika sa pangkalahatan ay mga tulay tungo sa pag-uunawaan, pakikiisa, pakikiramay - kapatiran at ugnayan.

\subsection{Ang Wika at Ang Kayamanan ng Daigdig}

Ang magising sa kayamanan ng wika ay magising sa kayamanan ng daigdig. Nabigkas noon ni Ferriols sa klase na ang iba't ibang wika ng mundo ay maihahambing sa samu't saring mga tanim. At tulad ng mga tanim, may kanya-kanyang bisa at pakinabang. May mga tanim na gamit na gamit ng tao. Ganito ang punong niyog. Bawa't bahagi ay kapaki-pakinabang; wala itong tapon. Ang bunga ay may tubig na maiinom at lamang makakain. Ang bunot ng bunga ay magagamit sa pagpapakintab ng sahig. Ang bao ay isinusuot ng sumasayaw ng manlalatik. Ang mga dahon ay tingting para sa walis at pawid para sa atip. Ang tangkay ay panggatong at naglalaman ng ubod na maaaring isahog sa lumpia. May mga tanim naman na tila baga hindi gaanong kapakipakinabang. Malay natin, mayroon palang halamang nagkukubli sa pinakakaloob-looban ng gubat sa Amazon o sa Aprika na, lingid sa kaalaman natin, nagtataglay ng lunas sa kanser o anumang malubhang sakit. Naghihintay lamang ito upang matuklasan at mapakinabangan. Parang ganito ang wika. Maraming tao ang hanga sa wikang Ingles dahil sa pakinabang nito lalo na sa kontekstong akademiko kung saan bihasa at komportable ang Ingles bilang wika ng mga nananaliksik, guro, estudyante, at maraming iba. Nakarating ang wikang ito sa ganyang kalagayan dahil sinubukan, hinasa, at sinanay hanapan ng tinig sa halos lahat ng mga larangang ginagalawan ng pamantasan. Dito nagmumula ang pananaw ng ilan ukol sa karukhaan ng Filipino kung kaya't hindi nabibigyan ng parehong pagkakataon ang Filipino na ito'y masanay at mahasa.

Inuulat ni Ferriols na ang isang bagay na ikinagugulat ng mga estudyante ay ang kayamanan ng wikang Filipino at ang mga hangganan ng wikang Ingles. Pareho rin lang namang mayaman at may hangganan. Sa pagsusumubok bigyan ng tinig ang pilosopiya sa Filipino, dito lamang natutuklasan ang kayamanan at hangganang ito. Sabi nga ni Ferriols ukol sa Griyego at Filipino:

${ }^{8}$ Ferriols, Sulyap, 115.

(c) 2018 Wilhelm Patrick Joseph S. Strebel https://www.kritike.org/journal/issue 22/strebel june2018.pdf ISSN 1908-7330

(c) $)$ BY-NC-ND 
O baka ganito ang sasabihin mo: "Kay hiwaga, kay yaman ng daigdig! Katotohanan man o aletheia, parehong pareho ang nararanasan, ibang iba ang nararanasan: nagkakaangkop, nagkakaiba, nagsasalubungan, naghihiwalayan, nakakahilo!" Tugon ko naman: nagugulumihanan ka. Nagtataka. Sinisimula mo na yatang kaibiganin ang karunungan. ${ }^{9}$

Alam ng sinuman ang tinutukoy ni Ferriols kung may karanasan siya ng pagtigil panandalian upang namnamin ang kayamanan ng daigdig sa kanyang karanasan sa panitikan. Hindi lamang mga salita ang kanyang binabasa. Inihahatid siya ng mga salita upang danasin ang kayamanan ng daigdig. Heto ang sinabi niya ukol sa pagtuturo sa kanya ni Dela Costa:

Pero pinamulat niya sa amin na ang daigdig ng mga makata ay lahat ng wika. Na ang tula ay bunga ng malalim na pagpasok, pagtanggap, at ligaya sa talagang totoo. Kailangan lamang ang matinding pagkagising. Dahil sa kanya, napansin namin na ang bawat salita ay may taglay na isip at damdamin.

Latin man o Ingles ang binabasa, hindi kami nakukulong sa letra ng libro. Nabubuhay ang mga letra at mga kataga at mga verso sa galíng ng pagpapamulat ni Horacio. Dahil sa kanya ang pumapaligid sa amin na katotohanan ay aming mas matinding nagigisnan. ${ }^{10}$

Tinatamaan ng puntong ito ni Ferriols ang katangian ng mahusay na guro. Anuman ang itinuturo ng guro, ang hamon sa kanya ay tulungan ang mga estudyante manahimik, dumanas, gumising. Kung nangyayari ito sa silidaralan, masasabi nating tunay ngang nabubuhay at natututo ang mga estudyante. Masuwerte ang mga tulad ni Ferriols na natuto mula sa mga tulad ni Dela Costa. Mapalad ang mga estudyante ni Ferriols na siyang pinasahan niya ng ganitong pagmumulat.

Ang hamon ngayon sa mga guro, lalo na ng balarila, panitikan, at pilosopiya ay ang gawain ng paggising sa mga estudyante na ang tunay na diwa ng mga tekstong binabasa nila ay lagpas sa mga pinag-aaralan nilang mga balangkas, gramatika, sistema, at teorya. Kung hindi nararanasan ng mga estudyante ang diwa na ito, hindi sila talaga nabubuhay o natututo.

${ }^{9}$ Roque J. Ferriols, Mga Sinaunang Griyego (Quezon City: Office of Research and Publications, 1992), 17.

${ }^{10}$ Ferriols, Sulyap, 121.

(c) 2018 Wilhelm Patrick Joseph S. Strebel https://www.kritike.org/journal/issue 22/strebel june2018.pdf ISSN 1908-7330 


\section{PILOSOPIYA NG WIKA NI FERRIOLS}

\subsection{Ang Sentro ng Wika Bilang Sentro ng Pagkamalikhain}

Inamin na natin na bawat wika ay may kakayahan at hangganan. Kung gayon, ang ginagawa ng malikhaing magsalita ay hindi naiiba sa pagpapanday: hinahango niya mula sa kalikasan ang mga materyales ng kanyang sining, kinikilala niya kung anu-ano ang maihahatid sa kanya ng bawat katangian ng mga materyales na ito at gagalawan na niyang lumikha ng bagong bagay mula dito.

Sa mga nag-aakala na 'taglish' (o 'enggalog,' biro ni Ferriols) ang solusyon sa mga hangganan ng Filipino, naghahain si Ferriols ng bagong perspektiba. Kung ang ibig sabihin ng 'taglish' ay ang walang pangingilatis na paghalili ng mga salitang Ingles sapagkat nalimutan ang katagang Filipino o di kaya'y ang pagpilit sa Filipino na sundan ang balangkas at ritmo ng Ingles, halata agad na walang husay o galing sa pagpapanday ang magaganap.

Mukhang ang modelo ni Ferriols ay ang mga makata tulad ni William Shakespeare. Sa pagpapanday ng wikang nakagisnan niya, ang wikang Ingles, ginawa niya ang pagpapayaman nito para sa kanyang mga berso at dula sa pamamagitan ng pakikitagpo sa Latin, Pranses, at Espanyol. Sa pakikitagpo na ito, yumaman at lumalim ang Ingles ngunit hindi ito napilitang ipagkasya ang sarili sa loob ng tiempo at ritmo ng ibang wika.

Kung kaya't may sentro pala ang wika. Dito madarama ang diwa at katutubong galing nito, at may pagkakataon para sa sinumang taong pasukin at damhin ang sentrong ito, kung hahayaan lamang niya ang kanyang sarili na maging tapat sa mga lumang tunog at bigkasin muli ang mga ito at matagpuan ang naliligaw niyang kalooban bilang bago, sa loob ng daigdig na palaging bago at tumutubo. ${ }^{11}$

May ibang nangyayari kung may bibigkas ng isang wika mula sa tunay nitong sentro at, mula sa sentrong iyon, lilikha siya ng mga bagong salita sa pamamagitan ng pagnanakaw (ang matatas magsalita, hindi nanghihiram bagkus nagnanakaw) mula sa ibang wika. ${ }^{12}$

May malikhaing pagnanakaw ng salita na siya namang nagpapalalim ng wika at karanasan sa taong marunong bumigkas. Dulot nito, lumalalim ang wika, tumatalas ang kakayahan nitong magpahayag ng kahulugan, at lumalalim din ang karanasan ng tao sa daigdig. Lumalawak ang mismong daigdig sa harap ng taong bumibigkas.

11 Ferriols, A Memoir, 222.

${ }^{12}$ Ibid., 219. Akin ang salin.

(c) 2018 Wilhelm Patrick Joseph S. Strebel https://www.kritike.org/journal/issue 22/strebel june2018.pdf ISSN 1908-7330

(c) BY-NC-ND 


\subsection{Ang Wika at Ang Danas-Daigdig ng Nagsasalita}

Noong kinuha si Danton Remoto bilang patnugot ng Office of Research and Publication, para sa paglimbag ng mga unang aklat ni Ferriols, hiniling ng huli na hawag babaguhin ng una ang paraan at estilo ng kanyang pagtatagalog. Mulat si Ferriols na iba siya magtagalog at para sa patnugot na gagalaw sa mga pamantayan ng Tagalog Bulakan, halimbawa, kailangang ibahin ang ilang mga parirala, baybay, at pagkabuo ng mga pangungusap ni Ferriols. Ang ilang mga halimbawa ay ang mga salita ni Ferriols tulad ng saktanin, pamamagit-an, at maari. Hilagang Samapalokenyo ang pabirong tawag ni Ferriols sa kanyang Tagalog. Noong bata pa daw siya sa Sampalok, Maynila, napansin niyang ang mga magulang niya at ang iba pang nakatanda sa kanya ay nakipag-usap sa isa't isa sa wikang Iloko. Kung kakausapin ng matatanda ang mga bata, tinatagalog sila. Sa murang edad napansin niyang iba siya magtagalog sa mga kababata niyang tubong Maynila ang mga magulang.

Nang walang pasintabi at pagpapaumanhin, pinasya ni Ferriols mamilosopiya sa wika na kanyang nagisnan: ang wika ng Hilagang Sampalok. Dito niya natuklasan ang kanyang sarili. Dito siya natuto dumanas at tumingin sa daigdig. Dito siya natuto sa kayamanan ng wika at ng daigdig kung kaya't sinasabi niya sa dulo ng A Memoir of Six Years,

Sa loob ng anim na taon, maaring matutunan na para sa makataong pag-iisip, mas mahusay ang Hilagang Sampalokenyo kaysa sa Griyego ni Platon. ${ }^{13}$

Hindi sinasabi ni Ferriols na mas dakila ang Filipino kaysa sa Griyego. Sinasabi lamang niya na ang katutubong wika ng taong namimilosopiya ay ang wikang pinaka-nababagay sa kanyang pagmumuni-muni. Sapagkat Griyego ang wikang nagisnan ni Platon, ang Griyego ang wikang pinakanababagay sa kanyang pag-iisip at pamumuhay. Sapagkat lumaki sa Hilagang Sampalok si Ferriols, ang wika ng Hilagang Sampalok ang pinakanababagay sa kanya.

Tinatamaan ni Ferriols sa puntong ito ang kahalagahan ng pagpapakatotoo. Sa harap ng daigdig, ng lipunan, ng kapwa tao na sumasalubong sa akin sa loob ng abot-tanaw ng sarili kong katutubong wika, ang pinaka-nababagay na tugon sa karanasang ito ay gagalaw at gagalaw pa rin sa loob ng abot-tanaw ng sarili kong katutubong wika. Kung sisikapin kong harapin ito sa wikang iba, di hamak, sa aking katutubong salita,

${ }^{13}$ Ibid., 222. Akin ang salin.

(c) 2018 Wilhelm Patrick Joseph S. Strebel https://www.kritike.org/journal/issue 22/strebel june2018.pdf ISSN 1908-7330 


\section{PILOSOPIYA NG WIKA NI FERRIOLS}

umaapak na ako palabas ng orihinal kong karanasan at lumalayo na ako sa daigdig na aking iniiralan mismo.

\section{Ang Ambag ng Pagsasanay sa mga Klasiko}

Kapag tinatanong si Ferriols kung paano siya napunta sa pilosopiya, palagi niyang sinasabi na inutusan siyang pag-aralan ito. At bilang huwarang Heswitang tumatalima sa kanyang mga superior, ginawa niya ito sa abot ng kanyang makakaya. Noong nagkita daw sila muli ni Kerr noong 1957, habang nag-aaral si Ferriols ng doktorado sa pilosopiya sa Pamantasang Fordham sa New York, at nalaman nito na pilosopiya ang ipinaaaral sa kanya, nasambit nito na sinasayang ng kanyang superior ang isang classical scholar. Pagkaraan pa ng ilang taon, sinasambit naman ni Ferriols na wala ring nasayang sapagkat ang kanyang pananahan sa mundo ng mga klasikong Griyego at Romano ay nakatulong sa kanyang pag-aaral at pagtuturo ng pilosopiya. Hindi matatanggihan ang halaga ng salin niya ng mga teksto ng mga sinaunang Griyego.

Bukod dito, mahihinuha rin bilang paglalagom sa pitong sulyap na ginawa ng papel na ito, na ang mahihiwatigan nating pilosopiya ng wika kay Ferriols ay nakulayan ng kanyang pagbabad sa mga klasiko. Sapagkat buhay para sa kanya ang mga salitang sinauna (buhay para sa kanya ang mga sinaunang manunulat, makata, o pilosopo), buhay din niyang naitatawid sa kanyang mga aklat at sa kanyang mga klase ang kayamanan ng mundo ng panitikan, at pilosopiya na siyang salamin ng kayamanan ng daigdig at ng kahulugan.

Sa huli, maaari ring ituring na sariwang alternatibo ang paraan ng pagmulat ni Ferriols sa wika. Malalim at malawak ang mga pagmumunimuni nina Wittgenstein, at ng buong analitikong tradisyon sa pamimilosopiya sa wika. Ngunit ang kanilang sistematiko, lohikal, at matematikong pagdulog sa paksa ay madaling maging pormalidad at anyo na lamang. Ang pilosopiya ng wika na nagsisimula at nagmumula sa punto de bista ng panitikan ay nagbibigay ng ibang larawan ng wika; kung paanong buhay na nililikha nito, kasama ang taong nagsusulat, bumibigkas at umuunawa ang buong mundo ng kahulugang maaari lamang makita kung bukas ang tao sa kalaliman at hiwaga ng daigdig.

Sa mga nakita nating mga puna ni Ferriols ukol sa wika, nagisnan din natin ang kalikasan ng pilosopiya; na ito ay isang gawain ng taong gumagalaw sa daigdig ng kapwa tao at nais pasukin ng mas masinsinan ang meron na ginagalawan nila ng kapwa tao. Ang mga bansag at tatak ay hindi sing-halaga at lalong hindi kapalit ng aktwal na karanasang pinagdaanan ng masusing pagsasagawa ng akto ng pamimilosopiya.

(c) 2018 Wilhelm Patrick Joseph S. Strebel

https://www.kritike.org/journal/issue 22/strebel june2018.pdf

ISSN 1908-7330

(c) $)$ BY-NC-ND 
Sa huli, inaanyayahan ng sulating ito ang iba pang mga nananaliksik na ipagpatuloy ang nasimulang landas ni Ferriols. Sa paninindigan niyang mamilosopiya sa wikang Filipino, nabigyan niya ang sarili niya ng pagkakataong magpakatotoo, aminin ang totoo at isagawa ang totoo. Kung ang pilosopiya nga ay pag-atupag sa katotohanan, ipinakita sa atin ni Ferriols ang daan.

Department of Philosophy, Ateneo de Manila University, Philippines

\section{References}

Ferriols, Roque J., “A Memoir of Six Years,” in Pagdiriwag sa Meron: A Festival of Thought Celebrating Roque J. Ferriols, S.J. (Quezon City: Office of Research and Publications, Ateneo de Manila University, 1997). Mga Sinaunang Griyego (Quezon City: Office of Research and Publications, Ateneo de Manila University, 1992).

Pambungad sa Metapisika (Quezon City: Office of Research and Publications, Ateneo de Manila Univeersity, 1991). Sulyap sa Aking Pinanggalingan, ed. by Leovino Ma. Garcia (Quezon City: Bughaw, Ateneo de Manila University Press, 2016). 